\title{
Iterative reconstruction of pulmonary MDCT angiography: Effects on image quality, effective dose and estimated organ dose to the breast
}

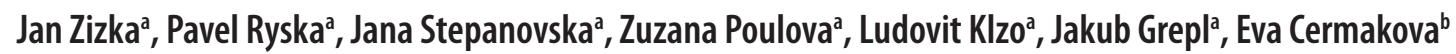

\begin{abstract}
Aims. To compare the image characteristics, effective dose and estimated organ dose to the female breast in pulmonary MDCT angiography (MDCTA), reconstructed with either standard filtered back projection (FBP), or iterative reconstruction in image space (IRIS).

Methods. Pulmonary MDCTA performed in 116 females (age 18 - 77 years; body mass index 15 - 48) was reconstructed with FBP $(n=52)$ or IRIS ( $n=64)$. Scans were acquired on a 128-row MDCT system using automatic tube current modulation, $100 \mathrm{kV}$ tube voltage, and a quality reference mAs value of 120 (FBP) and 80 (IRIS). Dose was calculated from CT dose index (CTDIvol) and dose length product (DLP) values utilising ImPACT software. Image noise was measured within the pulmonary artery. Qualitative visual assessment of the scans was performed ( $1=$ negligible noise, $5=$ noise obscuring diagnostic information).

Results. The average CTDIvol yielded $4.33 \mathrm{mGy}$ for FBP and $3.54 \mathrm{mGy}$ for IRIS, respectively (18.2\% decrease). The average effective scan dose was $2.73 \pm 0.57 \mathrm{mSv}$ (FBP) and $2.29 \pm 0.68 \mathrm{mSv}$ (IRIS), respectively ( $16.1 \%$ decrease). The estimated average organ dose to the breast decreased from 5.1 $\pm 1.1 \mathrm{mGy}$ (FBP) to $4.2 \pm 1.2 \mathrm{mGy}$ (IRIS, 17.6\% decrease). No nondiagnostic scans (score 5) were encountered in either group. Significant improvement in image noise levels $(P<0.01)$ and subjective image quality $(P<0.02)$ were noted in IRIS group.

Conclusion. Pulmonary MDCTA utilizing a $100 \mathrm{kV}$ technique, automatic tube current modulation, and iterative image reconstruction offers robust results in routine conditions among an unselected female population, with breast doses being comparable to two-view digital mammography. Moreover, iterative reconstruction offers improvements in both image noise and visual perception of the scans, thus suggesting a potential for further dose reduction.
\end{abstract}

Key words: multidetector computed tomography, pulmonary embolism, image reconstruction, iterative reconstruction, radiation effects, breast dose, effective dose

Received: May 26, 2013; Accepted: July 25, 2013; Available online: September 12, 2013

http://dx.doi.org/10.5507/bp.2013.059

${ }^{a}$ Department of Radiology, University Hospital Hradec Kralove, Hradec Kralove, Czech Republic

${ }^{b}$ Department of Biophysics, Faculty of Medicine in Hradec Kralove, Charles University in Prague, Hradec Kralove

Corresponding author: Jan Zizka, e-mail:jan.zizka@fnhk.cz

\section{INTRODUCTION}

Radiation dose resulting from medical exposure is continuously rising in the population. It has increased severalfold within the last three decades and, in some developed countries, e.g. the USA, it has reached the level of natural background radiation ${ }^{1}$. Computed tomography is the major contributor, accounting for more than two thirds of the entire cumulative medical exposure ${ }^{2}$.

To reverse this unfavourable trend, attributed mainly to the continuously increasing number of CT procedures, the adoption of adequate precautions is of utmost importance. Among the more promising approaches to dose reduction is the technique of iterative reconstruction of CT images which reduces radiation exposure by significantly supressing image noise $\mathrm{e}^{3,4}$.

The purpose of this study is to compare the image characteristics and effective and estimated organ dose to the female breast in pulmonary multidetector CT angiography (MDCTA) using both the standard filtered back projection (FBP) technique and iterative reconstruction in image space (IRIS). To our knowledge, no compara- tive study dealing with breast dose reduction in CTPA has been performed so far, although CTPA belongs to the frequently applied chest CT protocols. Additionally, a brief review of the literature covering the topics of effective and breast dose in pulmonary MDCTA as well as dose saving strategies is performed.

\section{MATERIALS AND METHODS}

A total of 150 consecutive adult females (age $18-77$ years, median 52 years) suspected of pulmonary embolism were prospectively enrolled in the study. The scans were reconstructed with standard FBP $(n=70)$ and, after CT reconstruction software upgrade, with IRIS $(n=80)$. The mean body mass index (BMI) in the FBP group equaled 25.3 (range 15.8 - 45.0) and in the IRIS group 27.9 (range $16.6-48.8$ ), the difference was not statistically significant $(P=0.13)$.

Scans were performed on a multidetector single source MDCT scanner from the level of the lung apex to the posterior costophrenic recesses with the following param- 
eters: collimation 64 × 2 × $0.6 \mathrm{~mm}$; tube voltage $100 \mathrm{kV}$; quality reference $\mathrm{mAs}$ value 120 (FBP) and 80 (IRIS); pitch factor of 1.4; rotation time of 0.3 seconds; reconstructed slice thickness $1 \mathrm{~mm}$; reconstruction kernel B20 (FBP) and I26 (IRIS). Automatic real time tube current modulation was switched on in order to balance image quality and radiation dose throughout the scans of subjects with widely variable attenuation profiles and BMI.

A contrast agent with a concentration of $400 \mathrm{mg} / \mathrm{mL}$ of iodine was injected by a dual head power injector with a flow rate of $4 \mathrm{~mL} / \mathrm{s}(3 \mathrm{~mL} / \mathrm{s}$ in rare cases of limited venous access quality). Subjects with body weight below $100 \mathrm{~kg}$ received $60 \mathrm{~mL}$ of contrast agent, subjects weighing $100 \mathrm{~kg}$ or more received $70 \mathrm{~mL}$. The bolus of contrast agent was followed by a $50 \mathrm{~mL}$ saline flush with the same flow rate. In accordance with local protocol, CTA acquisition was started automatically by using bolus tracking with a threshold set at 90 HU (subjects $<40$ years old with shorter circulation times), or $160 \mathrm{HU}$ (subjects $\geq 40$ years old), with a region of interest (ROI) placed within the pulmonary trunk.

Effective doses and estimated organ doses to the breast were calculated from the individually recorded CT dose index (CTDIvol, $\mathrm{mGy}$ ) and dose length product (DLP, mGy.cm) values utilizing ImPACT software (Impact, London).

Both FBP and IRIS scans were quantitatively assessed for adequate pulmonary artery enhancement and image noise by measuring the mean attenuation value and standard deviation of HU in a $2.5 \mathrm{~cm}^{2}$ ROI within the main pulmonary artery on a $1 \mathrm{~mm}$ slice.

Qualitative visual assessment of the scans was independently performed by two radiologists with 17 and 10 years of cross-sectional imaging experience respectively. The readers read the scans in a random fashion and were blinded to the type of CT image reconstruction, using a visual analogue scale:

$1=$ very low noise, optimal diagnostic quality

2 = low noise, good diagnostic quality

3 = increased noise, diagnostic quality

4 = high level noise, limited diagnostic quality

5 = unacceptable noise, non-diagnostic scan

Descriptive parameters of the study population and image reconstruction techniques were statistically compared using t-test or non-parametric tests (Mann-Whitney,
Kolmogorov-Smirnov). Qualitative parameters were compared using Fisher's exact test for contingency tables. Interobserver agreement between subjective image quality ratings was calculated by kappa reliability test with kappa $>0.75$ indicating substantial agreement. Statistical analysis was performed using NCSS 2007 programme (NCSS, Kaysville, Utah, USA) with $P$-values $<0.05$ considered as significant.

The institutional ethics committee waived the need for individual informed patient consent for this study as all examinations were performed as standard of care.

\section{RESULTS}

Of the 150 subjects enrolled into the study, 34 were excluded from evaluation due to deviation from the protocol (presence of metallic / respiratory / motion induced artifacts; upper extremities placed alongside the trunk; or contrast agent administration failure). Thus, a total of 52 FBP and 64 IRIS scans were available for evaluation.

The groups of FBP and IRIS scans showed no statistically significant differences when evaluating the mean attenuation values measured within the pulmonary artery (FBP: 358 HU; IRIS: $371 \mathrm{HU} ; P=0.66$ ); craniocaudal coverage of scans (FBP: $262 \mathrm{~mm}$; IRIS: $271 \mathrm{~mm} ; P=0.09$ ); or body mass index of the subjects.

The average CTDIvol yielded $4.33 \mathrm{mGy}$ for FBP (range 2.27 - 5.47) and $3.54 \mathrm{mGy}$ for IRIS (range 1.99 - 5.46) respectively (18.2\% decrease). The average DLP was $133.9 \mathrm{mGy} . \mathrm{cm}$ (FBP; range 75 - 193) and $115.9 \mathrm{mGy}$. cm (IRIS; range 56 - 195) respectively (13.4\% decrease).

The average effective dose was $2.73 \pm 0.57 \mathrm{mSv}$ (FBP; range 1.5 - 3.9) and $2.29 \pm 0.68 \mathrm{mSv}$ (IRIS; range $1.1-4.0$ ) respectively ( $16.1 \%$ decrease). The estimated average organ dose to the breast decreased from 5.1 $\pm 1.1 \mathrm{mGy}$ (FBP; range 2.7 - 6.6) to $4.2 \pm 1.2 \mathrm{mGy}$ (IRIS, range 2.4 - 6.7) respectively ( $17.6 \%$ decrease).

Additionally, image noise levels within the pulmonary artery were significantly lower $(P<0.001)$ in the IRIS group (median 18.9 HU) compared to the FBP group (median 23.1 HU).

A total of $28.1 \%$ of IRIS scans and $10.6 \%$ of FBP scans were qualitatively marked as optimal (score 1). In contrast, $16.3 \%$ of FBP scans and $3.1 \%$ of IRIS scans were

Table 1. Results of qualitative image analysis for readers 1 and 2.

\begin{tabular}{ccccc}
\hline \multirow{2}{*}{ Visual score } & \multicolumn{2}{c}{ FBP $(\mathrm{n}=52)$} & \multicolumn{2}{c}{ IRIS $(\mathrm{n}=64)$} \\
& Reader 1 & Reader 2 & Reader 1 & Reader 2 \\
\hline 1 & 6 & 5 & 18 & 25 \\
2 & 19 & 20 & 27 & 19 \\
3 & 19 & 18 & 17 & 2 \\
4 & 8 & 9 & 2 & 0 \\
5 & 0 & 0 & 0 & 18 \\
\hline
\end{tabular}

FBP = filtered back projection; IRIS = iterative reconstruction in image space. Visual scores: 1 = very low noise, optimal diagnostic quality; 2 = standard noise, good diagnostic quality; 3 = increased noise, diagnostic quality; 4 = high level noise, limited diagnostic quality; 5 = unacceptable noise, non-diagnostic scan. 

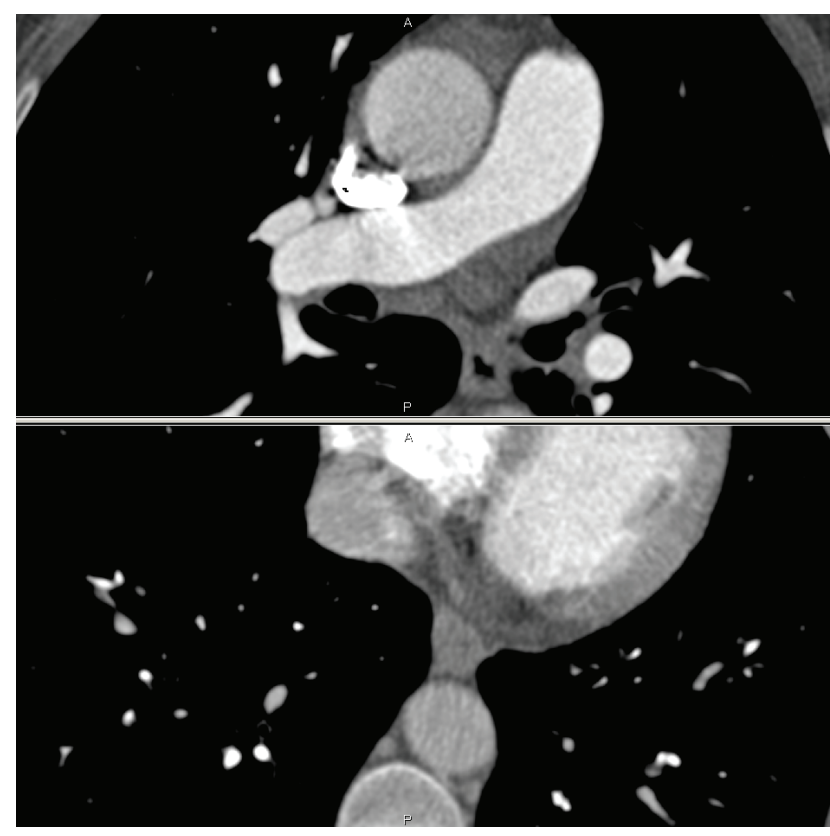

Fig. 1. FBP reconstructed $1 \mathrm{~mm}$ thin sections with optimal diagnostic quality (score 1). Very low image noise with homogenous vessel lumen opacity. Excellent delineation of segmental and subsegmental arterial branches.
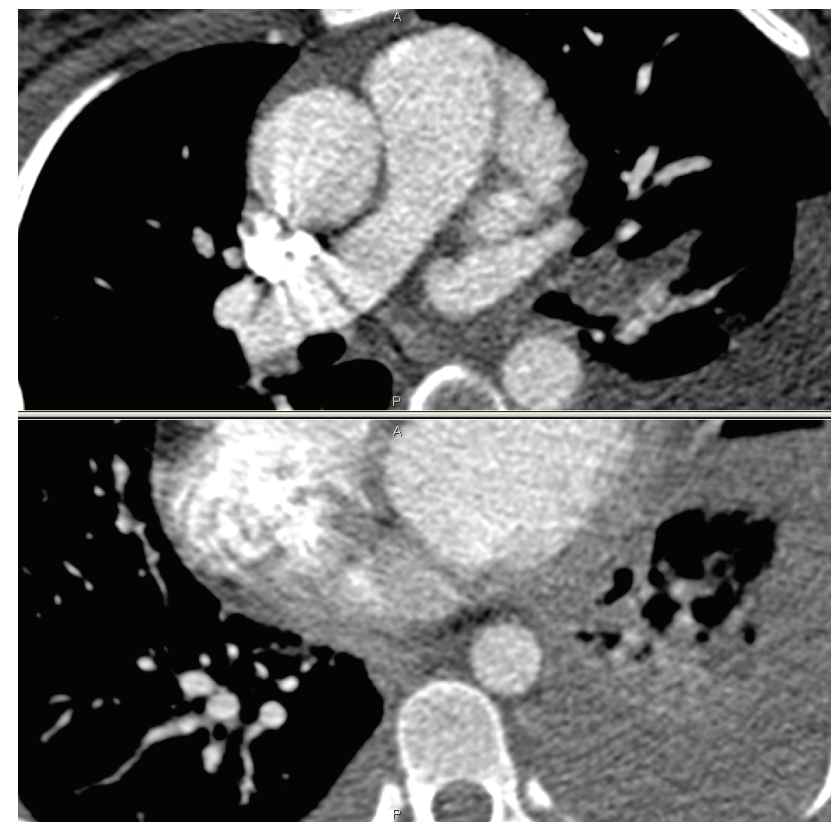

Fig. 3. FBP reconstructed $1 \mathrm{~mm}$ thin sections of limited diagnostic quality (score 4) in an obese subject with BMI of 30 High level of image noise with marked vessel lumen inhomogeneity which might simulate filling defects.

considered of limited diagnostic quality (score 4), see Figs. 1-4. No non-diagnostic scans (score 5) were noted in either group. Detailed image quality analysis results are shown in Table 1. According to Fisher's exact test, IRIS scans were rated significantly better in terms of image quality $(P=0.02)$.

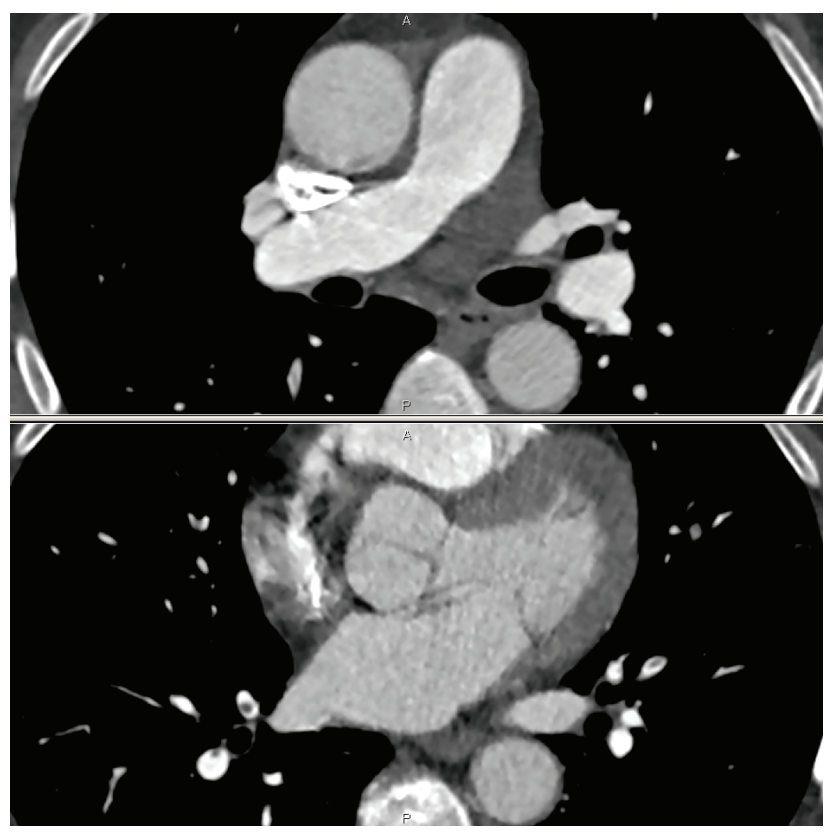

Fig. 2. IRIS reconstructed $1 \mathrm{~mm}$ thin sections with optimal diagnostic quality (score 1) in a patient with pulmonary embolism. Very low image noise with homogenous vessel lumen opacity. Excellent homogeneity and delineation of segmental and subsegmental arterial branches, convincing evidence of multiple non-occlusive subsegmental emboli.

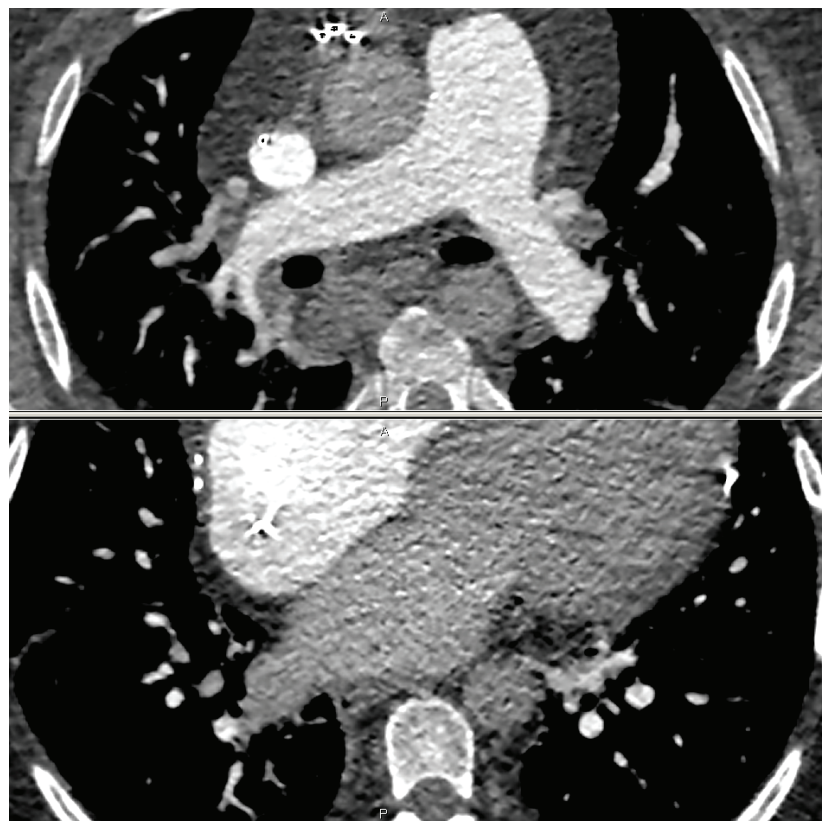

Fig. 4. IRIS reconstructed $1 \mathrm{~mm}$ thin sections of limited diagnostic quality (score 4) in an obese subject with BMI of 38 . High level of image noise with marked vessel lumen inhomogeneity which might simulate filling defects.

The agreement between the two observers was strong (kappa=0.85; kappa's standard error=0.07). 


\section{DISCUSSION}

With the almost exponential rise in the number of CT procedures performed worldwide annually, the need for the standardization, optimization, and stringent adherence to ALARA ("as low as reasonably achievable") principles is stronger than ever. Nonetheless, even recent publications have indicated that there is a substantial variance in doses applied for the same types of CT procedures at different institutions, as well as there being significant potential for reducing radiation dose in routine CT examinations ${ }^{5,6}$. A multi-centric study from California estimates that one in 330 females undergoing MDCTA for ruling out pulmonary embolism at the age of twenty will develop a radiation induced cancer ${ }^{5}$. This study also highlights a significant variance in the effective doses applied in pulmonary MDCTAs among four institutions: median of $10 \mathrm{mSv}$, range $2-30 \mathrm{mSv}\left(\right.$ ref. $\left.^{5}\right)$.

Iterative reconstruction of $\mathrm{CT}$ image offers significant image noise reduction when compared to FBP, thus allowing $\mathrm{CT}$ acquisitions of comparable image quality with lower radiation dose. The advantages of iterative image reconstruction for dose reduction in chest CT studies were recently demonstrated in few innovative studies ${ }^{3,4,7,8}$.

The average effective dose in our unselected population of females (with BMI widely ranging from 15 to 48 ) was $2.73 \mathrm{mSv}$ in the FBP group, i.e. at the lower end of the dose ranges demonstrated in the multi-institutional studies $^{5,6,9}$. Thus, it can be inferred that our local protocols were optimized in accordance with ALARA principles.

Our results show that even in the settings of optimized FBP based CT protocols, both image quality improvements and radiation dose reductions can be achieved simultaneously by applying techniques of iterative reconstruction which, in our population, further reduced the average effective dose by $16.1 \%$ to $2.29 \mathrm{mSv}$. Similarly, the estimated organ dose applied to the female breast decreased by $17.6 \%$ to $4.2 \mathrm{mGy}$ in the IRIS group, without inducing streak artifacts reported when using bismuth breast shielding ${ }^{10,11}$ and without compromising objective or subjective image quality. This desirable radiation reducing effect is amplified when given the significant proportion of young females undergoing pulmonary MDCTA; overall breast tissue radiosensitivity; as well as the fact that the breast is exposed to the primary beam in virtually all studies.

To our knowledge, this is the first study to evaluate the potential benefit of iterative reconstruction of pulmonary MDCTA in terms of quantifying estimated organ dose applied to the female breast. Our results show that iterative reconstruction enables further reduction of both effective and breast organ dose in this procedure. Moreover, iterative reconstruction offers improvements in image noise and visual perception of the scans with reduced radiation dose, thus offering potential for further dose reduction: in our study, optimal scan quality was noted more than 2.5 times more frequently in the IRIS group than in the FBP group.

Generally, there are many ways of reduing radiation dose in chest CT studies. Fortunately, most of these can be combined in order to boost their synergic effect. The most important approaches include: lowering the kilovoltage and/or tube current time product; partial scanning; high pitch scanning; automatic tube current modulation; or using bismuth shielding for the radiosensitive breast. Applying the techniques of iterative image reconstruction can also be added to this list.

There are two major reasons why reducing tube kilovoltage in pulmonary CT angiography significantly reduces radiation dose. First, with lowered kilovoltage there is more than a linear decrease in radiation dose: when the tube voltage is reduced from 120 to $100 \mathrm{kV}$, the effective dose decreases by $45 \%$ (ref. ${ }^{12}$ ). Second, lowering the kilovoltage is particularly suitable for CT angiographic studies because $\mathrm{x}$-ray absorption of iodine increases as the effective energy of the x-ray beam decrease ${ }^{13}$, thus enhancing the image contrast between the iodine filled vascular lumen and other structures, including thrombi. Furthermore, the chest is particularly suitable for lowdose CT protocols because of its low x-ray absorption (due to the abundance of air) and the inherent high contrast between vascular structures and the surrounding air. Owing to the high heat capacity of the x-ray tube used, we were able to use $100 \mathrm{kV}$ tube voltage in all patients including those subjects with a BMI > 40 which allowed us to keep the radiation dose low even in very obese subjects. Our results are in agreement with Schueller-Weidekamm et al. ${ }^{14}$ who observed a $65 \%$ dose reduction in pulmonary CTA when lowering the tube voltage from 140 to $100 \mathrm{kV}$, while maintaining comparable image quality. Notably, a multi-institutional survey among fifteen different pulmonary MDCTA protocols ${ }^{6}$ showed considerable variations in the peak kilovoltage and miliampere-seconds used: eleven sites routinely used $120 \mathrm{kV}$ and four sites $140 \mathrm{kV}$ protocols, even in low BMI subjects; no breast dose measurements were provided. Also, in a study by Parker et al. ${ }^{15}$ tube voltage was set to $140 \mathrm{kV}$ in all subjects, leading to a high mean breast dose of $20 \mathrm{mGy}$.

There is a linear correlation between radiation dose and tube current time product (mAs). Therefore, a cautious selection of appropriately low miliampere-second values is also crucial for keeping the radiation dose as low as possible. However, it is impractical to manually adapt these parameters correctly to actual scanning conditions (wide range of BMIs, position of upper extremities during scan, presence of metallic implants etc.). In addition, inappropriately low $\mathrm{mAs}$ settings can lead to an unacceptable increase of image noise. Therefore, it is advisable to set a proper reference $m A s$ value for a standard patient and utilize automatic tube current modulation which adjusts the acutal $\mathrm{mA}$ value in real time depending on the attenuation as a function of the projection angle and/or overall attenuation of the actual slice. Vollmar et al. ${ }^{10}$ reported a $10 \%$ reduction of dose to the breasts when using automatic tube current modulation in thoracic $\mathrm{CT}$ studies. According to our local protocol, we routinely utilized automatic real time modulation of the tube current in $\mathrm{x}-\mathrm{y}$, , and $\mathrm{z}$-axis in order to maximize dose reductions and minimize the risk of excessive increases in image noise. 
Partial CT scan and organ based tube current modulation are alternative techniques which selectively reduce dose to particular organs, e.g. breasts. The x-ray tube is switched into full power mode during only approx. $240^{\circ}$ of the $360^{\circ}$ rotation. As the radiaton is switched off (partial CT scan), or significantly lowered (organ based tube current modulation), when the tube is anterior to the patient, selective lowering of the absorbed dose to anteriorly located organs (e.g. breast or eye lens) can be achieved. However, in order to achieve the same level of exposure and image noise, the tube current from posterior projections must be proportionally increased which leads to an increase in dose to the spine (bone marrow). In a study on a semi-anthropomorphic phantom, Vollmar et al. ${ }^{10}$ reported a $45 \%$ breast dose reduction with this technique, however, the spinal organ dose increased by $19 \%$. Similar results were reported with the organ based tube current modulation technique, yielding $12 \%$ to $39 \%$ breast dose reduction, based on the size of thorax phantom $^{11}$. Partial CT scanning technique was not available on our scanner.

Recently, a study on radiation dose in pulmonary dual source / dual energy pulmonary MDCTA showed that novel techniques of high pitch scanning may also significantly lower radiation ${ }^{16}$. At $100 \mathrm{kV}$, CTDIvol was 3.90 mGy for a high-pitch dual source CT scan compared to $5.97 \mathrm{mGy}$ for a standard single source CT scan. No organ or effective dose measurements were provided.

There is some controversy over using bismuth or tungsten-antimony shielding of the breast tissue in chest CT. Although some authors advocate their use owing to their significant breast dose reducing effect ${ }^{9,17,18}$, other studies referred to increased noise level, decreased image quality, and the presence of streak and beam hardening artifacts induced by the shields ${ }^{10,11}$. Consequently, the American Association of Physicists in Medicine recently issued a statement on the use of bismuth shielding in CT scanning which makes note of several disadvantages to their use. These include: induction of streak and beam hardening artifacts; unpredictable deviation from desired dose levels and image quality when used in conjunction with automatic tube current modulation; and wasting some of the patient's radiation exposure without contributing to image formation ${ }^{19}$. According to this statement, alternative approaches for reducing breast dose other than organ shields should be preferred. No breast shielding is therefore used at our institution, including our study cohort.

Taking into account that the radiosensitivity of breast tissue is relatively high, particularly in young, pregnant, and lactating females, it is surprising that there is limited literature on organ dose to the female breast associated with pulmonary CT angiography studies, despite the fact that many scanned females are of reproductive age. In a study of 1,325 pulmonary CT angiographies, 797 were performed in female patients (60\%); of these, $26.7 \%$ were performed in females younger than 35 years old ${ }^{15}$.

Given the vast variety of protocols, techniques, and (if implemented) dose reduction strategies published in the literature, the dose ranges for routine pulmonary MDCTA vary substantially. Organ doses to the breast ranged from 3.9 to $20 \mathrm{mGy}^{15,20,21}$. However, in the study of Hurwitz et al. ${ }^{18}$, on $120 \mathrm{kV}$ and $140 \mathrm{kV}$ protocols, large anthropomorphic phantom measurements showed average breast doses of $44 \mathrm{mGy}$ at $120 \mathrm{kV}$ and even $62 \mathrm{mGy}$ when scanning at $140 \mathrm{kV}$ (without breast shielding). For comparison, two-view digital mammography and screen-film mammography involve average mean glandular radiation doses of 3.7 and $4.7 \mathrm{mGy}$, respectively ${ }^{22}$. Our results show that pulmonary MDCTA can routinely be performed with average breast dose being fully comparable with those of two-view mammography (4.2 mGy in our study population).

Mean effective dose in pulmonary MDCTA from the literature ranges from 2 to $30 \mathrm{mSv}$, depending on protocol and method of measurement ${ }^{5,20,23,24}$. Effective dose achieved with our protocol $(2.3 \mathrm{mSv})$ is at the very low end of this range.

Even so, there are serious implications for possible cancer induction in body CT protocols. In the study of Hurwitz et al. ${ }^{20}$, the lifetime attributable risk of breast or lung cancer in females undergoing a single multidetector pulmonary CTA examination was estimated to be 106 cases of cancer attributable to radiation exposure per 100000 subjects exposed at the age of 55 years. The implications for cancer induction in young women groups are more substantial: the rate increases to 251 cases of cancer per 100000 females exposed at the age of 25 years. Therefore, every effort should be made to lower the radiation dose in order to minimize the risks of cancer induction, mainly in young and peripartum women. Iterative reconstruction can significantly aid in achieving this goal.

\section{CONCLUSION}

Our results show that a CTPA protocol combining $100 \mathrm{kV}$ technique, automatic tube current modulation and iterative image reconstruction is capable of a substantial reduction of both breast dose (4.2 $\mathrm{mGy}$ in our study) and effective dose $(2.3 \mathrm{mSv})$. These values achieved are at the very low end of the dose ranges published ${ }^{5,18,20,21}$ despite being obtained in routine clinical settings and in an unselected population with a wide range of BMI, spanning from 15.8 to 48.8 . Additionaly, the lower radiation dose achieved with iterative image reconstruction was associated with a significantly better objective and subjective image quality than standard filtered back projection, suggesting possible further dose reduction. This would need more research.

\section{ACKNOWLEDGMENTS}

The authors thank Sean Kearns (Dumfries and Galloway Royal Infirmary, Dumfries, United Kingdom) for his valuable comments and careful revision of the manuscript. 


\section{CONFLICT OF INTEREST STATEMENT}

The authors state that there are no conflicts of interest regarding the publication of this article.

\section{REFERENCES}

1. Mettler FA Jr, Bhargavan M, Faulkner K, Gilley DB, Gray JE, Ibbott GS, Lipoti JA, Mahesh M, McCrohan JL, Stabin MG, Thomadsen BR Yoshizumi TT. Radiologic and nuclear medicine studies in the United States and worldwide: frequency, radiation dose, and comparison with other radiation sources 1950-2007. Radiology 2009;253:520-31.

2. Kalra MK, Maher MM, Toth TL, Hamberg LM, Blake MA, Shepard JA, Saini S. Strategies for $C T$ radiation dose optimization. Radiology 2004;230:619-28.

3. Leipsic J, Labounty TM, Heilbron B, Min JK, Mancini GB, Lin FY, Taylor C, Dunning A, Earls JP. Estimated radiation dose reduction using adaptive statistical iterative reconstruction in coronary CT angiography: the ERASIR study. Am J Roentgenol 2010;195:655-60.

4. Pontana F, Pagniez J, Flohr T, Faivre JB, Duhamel A, Remy J, RemyJardin M. Chest computed tomography using iterative reconstruction vs filtered back projection (Part 1): evaluation of image noise reduction in 32 patients. Eur Radiol 2011;21:636-43.

5. Smith-Bindman R, Lipson J, Marcus R, Kim KP, Mahesh M, Gould R Berrington de González A, Miglioretti DL. Radiation dose associated with common computed tomography examinations and the associated lifetime attributable risk of cancer. Arch Intern Med 2009; 169:2078-86.

6. Johnson PT, Naidich D, Fishman EK. MDCT for suspected pulmonary embolism: multi-institutional survey of 16-MDCT data acquisition protocols. Emerg Radiol 2007;13(5):243-9.

7. Pontana F, Duhamel A, Pagniez J, Flohr T, Faivre JB, Hachulla AL, Remy J, Remy-Jardin M. Chest computed tomography using iterative reconstruction vs filtered back projection (Part 2): image quality of low-dose CT examinations in 80 patients. Eur Radiol 2011;21(3):636 43.

8. Prakash P, Kalra MK, Digumarthy SR, Hsieh J, Pien H, Singh S, Gilman MD, Shepard JA. Radiation dose reduction with chest computed tomography using adaptive statistical iterative reconstruction technique: initial experience. J Comput Assist Tomogr 2010;34(1):40-5.

9. Parker MS, Kelleher NM, Hoots JA, Chung JK, Fatouros PP, Benedict $\mathrm{SH}$. Absorbed radiation dose of the female breast during diagnostic multidetector chest $\mathrm{CT}$ and dose reduction with a tungstenantimony composite breast shield: preliminary results. Clin Radio 2008;63(3):278-88.

10. Vollmar SV, Kalender WA. Reduction of dose to the female breast in thoracic CT: a comparison of standard-protocol, bismuth-shielded, partial and tube-current-modulated CT examinations. Eur Radiol 2008;18(8):1674-82.

11. Wang J, Duan X, Christner JA, Leng S, Yu L, McCollough CH. Radiation dose reduction to the breast in thoracic $\mathrm{CT}$ : comparison of bismuth shielding, organ-based tube current modulation, and use of a globally decreased tube current. Med Phys 2011;38(11):6084-92.

12. Björkdahl P, Nyman U. Using 100- instead of $120-k V p$ computed tomography to diagnose pulmonary embolism almost halves the radiation dose with preserved diagnostic quality. Acta Radiol 2010;51(3):260-70

13. Sigal-Cinqualbre AB, Hennequin R, Abada HT, Chen X, Paul JF. Lowkilovoltage multi-detector row chest $\mathrm{CT}$ in adults: feasibility and effect on image quality and iodine dose. Radiology 2004;231(1):16974.

14. Schueller-Weidekamm C, Schaefer-Prokop CM, Weber M, Herold CJ, Prokop M. CT angiography of pulmonary arteries to detect pulmonary embolism: improvement of vascular enhancement with low kilovoltage settings. Radiology 2006;241(3):899-907.

15. Parker MS, Hui FK, Camacho MA, Chung JK, Broga DW, Sethi NN. Female breast radiation exposure during $\mathrm{CT}$ pulmonary angiography. AJR Am J Roentgenol 2005;185(5):1228-33.

16. De Zordo T, von Lutterotti K, Dejaco C, Soegner PF, Frank R, Aigner F, Klauser AS, Pechlaner C, Schoepf UJ, Jaschke WR, Feuchtner GM. Comparison of image quality and radiation dose of different pulmonary CTA protocols on a 128-slice CT: high-pitch dual source CT, dual energy CT and conventional spiral CT. Eur Radiol 2012;22(2):279-86.

17. Yilmaz MH, Albayram S, Yașar D, Ozer H, Adaletli I, Selçuk D, Akman C, Altuğ A. Female breast radiation exposure during thorax multidetector computed tomography and the effectiveness of bismuth breast shield to reduce breast radiation dose. J Comput Assist Tomogr 2007;31(1):138-42.

18. Hurwitz LM, Yoshizumi TT, Goodman PC, Nelson RC, Toncheva G, Nguyen GB, Lowry $C$, Anderson-Evans $C$. Radiation dose savings for adult pulmonary embolus 64-MDCT using bismuth breast shields, lower peak kilovoltage, and automatic tube current modulation. AJR Am J Roentgenol 2009;192(1):244-53.

19. The American Association of Physicists in Medicine. AAPM Position Statement on the Use of Bismuth Shielding for the Purpose of Dose Reduction in CT scanning. Published February 7, 2012. Available at: http://www.aapm.org/publicgeneral/BismuthShielding.pdf

20. Hurwitz LM, Reiman RE, Yoshizumi TT, Goodman PC, Toncheva G, Nguyen G, Lowry C. Radiation dose from contemporary cardiothoracic multidetector $\mathrm{CT}$ protocols with an anthropomorphic female phantom: implications for cancer induction. Radiology 2007;245(3):742-50.

21. Litmanovich D, Tack D, Lin PJ, Boiselle PM, Raptopoulos V, Bankier AA. Female breast, lung, and pelvic organ radiation from dose-reduced 64-MDCT thoracic examination protocols: a phantom study. AJR Am J Roentgenol 2011;197(4):929-34.

22. Hendrick RE. Radiation Doses and Cancer Risks from Breast Imaging Studies. Radiology 2010:257(1):246-53.

23. Kuiper JW, Geleijns J, Matheijssen NA, Teeuwisse W, Pattynama PM. Radiation exposure of multi-row detector spiral computed tomography of the pulmonary arteries: comparison with digital subtraction pulmonary angiography. Eur Radiol 2003;13(7):1496-1500.

24. Woo JK, Chiu RY, Thakur Y, Mayo JR. Risk-benefit analysis of pulmonary $C T$ angiography in patients with suspected pulmonary embolus. AJR Am J Roentgenol 2012;198(6):1332-9. 